\section{(A) Check for updates}

Cite this: Food Funct., 2021, 12, 8507

\title{
Human milk oligosaccharide 2' -fucosyllactose supplementation improves gut barrier function and signaling in the vagal afferent pathway in mice $\uparrow$
}

\author{
Sunhye Lee, $t^{\mathrm{a}}$ Michael L. Goodson, (D) ${ }^{a}$ Wendie Vang, ${ }^{a}$ Jennifer Rutkowsky, (D) ${ }^{\mathrm{b}}$ \\ Karen Kalanetra, ${ }^{\mathrm{C}}$ Mrittika Bhattacharya, ${ }^{\mathrm{C}}$ Daniela Barile (iD) ${ }^{\mathrm{C}}$ and \\ Helen E. Raybould (iD a
}

\begin{abstract}
$2^{\prime}$-Fucosyllactose $\left(2^{\prime}-\mathrm{FL}\right)$ is one of the predominant oligosaccharides found in human milk and has several well-established beneficial effects in the host. It has previously been shown that 2'-FL can improve the metabolic phenotype in high-fat (HF)-fed mice. Here we investigated whether dietary supplementation with 2'-FL was associated with improved intestinal barrier integrity, signaling in the vagal afferent pathway and cognitive function. Mice were fed either a low-fat (LF, 10\% fat per kcal) or HF (45\% fat per kcal) diet with or without supplementation of $2^{\prime}-\mathrm{FL}(10 \% \mathrm{w} / \mathrm{w})$ in the diet for 8 weeks. Body weight, energy intake, fat and lean mass, intestinal permeability (ex vivo in Ussing chambers), lipid profiles, gut microbiome and microbial metabolites, and cognitive functions were measured. Vagal afferent activity was measured via immunohistochemical detection of c-Fos protein in the brainstem in response to peripheral administration of cholecystokinin (CCK). 2'-FL significantly attenuated the HF-induced increase in fat mass and energy intake. 2'-FL significantly reduced intestinal permeability and significantly increased expression of interleukin (IL)-22, a cytokine known for its protective role in the intestine. Additionally, 2'-FL led to changes in the gut microbiota composition and in the associated microbial metabolites. Signaling in the vagal afferent pathway was improved but there was no effect on cognitive function. In conclusion, 2'-FL supplementation improved the metabolic profiles, gut barrier integrity, lipid metabolism and signaling in the vagal afferent pathway. These findings support the utility of 2'-FL in the control of gut barrier function and metabolic homeostasis under a metabolic challenge.
\end{abstract}

Received 2nd March 2021 Accepted 17th June 2021 DOI: $10.1039 / \mathrm{d} 1 \mathrm{fo} 00658 \mathrm{~d}$ rsc.li/food-function or contributing role for the microbiota in multiple pathologies. ${ }^{2,3}$ Thus, manipulation of the gut microbiota to treat metabolic and cognitive impairments associated with dysbiosis is an attractive peripheral target. ${ }^{4-6}$ In particular, the use of non-invasive approaches such as the use of prebiotics to change the microbiota has been of particular interest. As metabolic substrates for beneficial bacteria, prebiotics have been shown to exert metabolic and cognitive benefits in association with compositional changes in the gut microbiota and their metabolites in the host. ${ }^{5,7-14}$

Human milk oligosaccharides (HMO) have been shown to have multi-functional, health-promoting activities and are consequently gaining considerable interest in the infant formula industry as well as for prebiotic applications in adults. ${ }^{15} \mathrm{~A}$ handful of HMO are being produced industrially using recombinant methodology and supplementation of oligosaccharides such as 2 -fucosyllactose (2'-FL) and lacto- $N$-neotetraose (LNnT) to infant formula is now possible. $2^{\prime}$-FL is a predominant oligosaccharide in human milk. ${ }^{16}$ Administration of $2^{\prime}$-FL 
can act as a prebiotic to improve gut dysbiosis. ${ }^{17-19} 2^{\prime}$-FL also has direct beneficial effects on the host at the level of the gastrointestinal tract to improve intestinal immune and barrier function as shown in in vitro studies and following dietary supplementation in vivo. ${ }^{20,21}$ Another potentially important, yet poorly understood, beneficial function of HMO is their contribution to the cognitive development of infants. Dietary supplementation with 2 '-FL has been shown to improve cognitive function in rodents. ${ }^{22} 2$-FL enhanced hippocampal long-term potentiation in vitro suggesting a direct effect on neurons in the central nervous system consistent with increased memory consolidation seen in rodent studies. ${ }^{23}$ The ability of $2^{\prime}$-FL to influence cognitive function and improve learning and memory has also been shown to involve a vagally-mediated pathway. $^{24}$

We have previously shown that administration of 2 '-FL in the drinking water has beneficial metabolic effects in mice fed a high-fat (HF) diet, including decreased body weight and adiposity, decreased gut and adipose tissue inflammation and drove significant changes in the composition of the gut microbiota and its metabolites. ${ }^{7}$ However, whether the beneficial metabolic effects of 2 '-FL were associated with changes in intestinal permeability was not investigated in the previous study. There is evidence to suggest that HF diet-induced obesity is driven by changes in gut permeability with the consequent translocation of deleterious bacterial metabolites into the circulation. ${ }^{25}$ These bacterial metabolites can act systemically to induce inflammatory responses in metabolically-active tissues and also impair the vagal afferent signaling. ${ }^{26,27}$ Hyperphagia in response to $\mathrm{HF}$ feeding is associated with decreased activation of the vagal afferent pathway to intestinal satiety factors, in particular cholecystokinin (CCK); changes in gut microbial metabolites or improved gut permeability may help restore vagally-mediated intestinal satiety responses and decrease food intake. Thus, this study investigated the effects of 2'-FL supplementation in the diet on metabolism, the vagal afferent pathway and cognitive function in mice with or without the metabolic challenge of HF feeding. Changes in general phenotypes (body weight, energy intake, body composition), gut barrier integrity, lipid profiles, cecal microbiome and metabolites, and cognitive behaviors were measured. We also determined the effect of 2 '-FL on the vagal afferent pathway.

\section{Methods and materials}

\section{Animals and diets}

Animals were maintained and experiments performed in compliance with institutional guidelines. All procedures were performed in accordance to protocols approved by the Institutional Animal Care and Use Committee (\# 21900, University of California, Davis). Male C57BL/6J mice ( $n=32,8$ / group; 6-week-old, JAX, Sacramento, CA) were split into 4 weight-matched groups and fed either a low-fat (LF/CON; 10\% kcal as fat), high-fat ( $\mathrm{HF} / \mathrm{CON} ; 45 \% \mathrm{kcal}$ as fat), or $\mathrm{LF}$ or $\mathrm{HF}$ diet with 2'-FL (LF/2'-FL or $\mathrm{HF} / 2^{\prime}-\mathrm{FL}$; provided by BASF, Ludwigshafen, Germany; batch no. 012644-L 04) at 10\% (w/w) in diet for 8 weeks (Table S1†). All animals were housed individually at $22.6 \pm 0.6{ }^{\circ} \mathrm{C}, 59.2 \pm 8.5 \%$ relative humidity (mean \pm standard deviation) with $12 \mathrm{~h}: 12 \mathrm{~h}$ light-dark cycles. Body weight and food and water intake were measured once a week. Food efficiency was determined as weight gain (g)/energy intake (kcal). Body composition for fat and lean mass was analyzed in live animals using EchoMRI-100 TM from Echo Medical Systems (Houston, TX). After 8 weeks on respective diets, mice were fasted for $4 \mathrm{~h}$, prior to administration of CCK (IP; $20 \mu \mathrm{g} \mathrm{kg}^{-1}$; Bachem Americas, Torrance, CA) $2 \mathrm{~h}$ before euthanasia to determine c-Fos expression in hindbrain, and euthanized using deep anesthesia induced with pentobarbital (Fatal Plus, Vortech Pharmaceuticals, Dearborn, MI; 300 mg $\mathrm{kg}^{-1}$; i.p.). Blood was collected via cardiac puncture in $\mathrm{K} 3-$ EDTA tubes and centrifuged at $1000 \mathrm{~g}$ for $10 \mathrm{~min}$ at $4{ }^{\circ} \mathrm{C}$ to obtain plasma. The liver, ileum, cecum and contents, colon, visceral fat pads, nodose ganglion, and brain were collected. Plasma and tissues were snap-frozen and stored at $-80{ }^{\circ} \mathrm{C}$ until analysis.

\section{Cognitive assessment}

Cognitive assessments were performed after 7 weeks on the respective diets during the light period.

Y-maze test. Short-term working memory was assessed by the Y-maze spontaneous alternation test. Mice were adapted to the testing room for $30 \mathrm{~min}$ then placed in the center of the Y-maze and were tracked with an overhead camera for the extent of a 6 min trial. An elevated white plastic Y-maze with three $35 \mathrm{~cm}$ arms at 120-degree angles was used. The number of entries into each arm was recorded and scored with Ethovision XT videotracking software (Version 14.0, Noldus Information Technologies, Leesburg, VA). Alternation score was computed as the number of times the three arms were sequentially entered. The \% alternation score is the number of alternations divided by the total number of arms entered.

Open field test. General locomotor activity levels, anxiety, and willingness to explore were assessed by the open field test. Mice were adapted to the testing room for $60 \mathrm{~min}$ then placed in the center of a white plastic box $(40 \times 40 \times 40 \mathrm{~cm})$ and were tracked with an overhead camera for the extent of a $15 \mathrm{~min}$ trial with Ethovision XT videotracking software (Version 14.0, Noldus Information Technologies, Leesburg, VA). Perimeter was defined as the outer $10 \mathrm{~cm}$ region of the $40 \mathrm{~cm}$ box. Time (second) spent in the center or perimeter zones of the open field was determined.

Novel object recognition test. Short-term non-associative memory was assessed in the novel object test based on the natural exploration of novelty in mice. Day 1 consisted of a 15 min environmental habituation, which also served as our open field test. Day 2 consisted of a $12 \mathrm{~min}$ familiarization period with two identical objects (plastic flasks or cones) and a 12 min test with one known and one novel object (one flask and one cone). Mice, adapted to testing room for $60 \mathrm{~min}$, were placed in the center of a white plastic box $(40 \times 40 \times 40 \mathrm{~cm})$ 
and were tracked with an overhead camera. The familiarization period and novel object test were performed $4 \mathrm{~h}$ apart. The time spent at both the novel and known objects were recorded with EthoVision XT video tracking software (Version 14.0, Noldus Information Technologies, Leesburg, VA). Discrimination index was calculated as difference in exploration time for familiar object, but then dividing this value by the total amount of exploration of the novel and familiar objects $[\mathrm{DI}=(\mathrm{TN}-\mathrm{TF}) /(\mathrm{TN}+\mathrm{TF})]$.

\section{Oral glucose tolerance test (OGTT)}

Mice were food-deprived for 6 hours and orally gavaged with a glucose solution ( $2 \mathrm{~g}$ per $\mathrm{kg}$ body weight using 50\% dextrose solution [Dextrose Injection, USP, Hospira. Lake Forest, IL]). Glycemia was measured by using a glucometer (True Metrix, Tuvidia Health, Ft. Lauderdale, FL) before (0 min) and after $(15,30,60,90$, and $120 \mathrm{~min})$ glucose administration. ${ }^{28}$ OGTTs were performed 2 days after cognitive assessments.

\section{Barrier function assessment}

At necropsy, a section of cecum was opened along the mesenteric border and mounted in Ussing chambers (Physiologic Instruments, San Diego, CA), exposing $0.1 \mathrm{~cm}^{2}$ of tissue surface area to $2.5 \mathrm{ml}$ of oxygenated Krebs-glucose (10 mM) and Krebs-mannitol $(10 \mathrm{mM})$ at $37{ }^{\circ} \mathrm{C}$ on the serosal and luminal sides, respectively. The paracellular pathway and transcellular pathway were measured as the flux of fluorescein isothiocyanate conjugated to $4 \mathrm{kDa}$ dextran (FD-4; SigmaAldrich) and horseradish peroxidase (HRP Type VI; Sigma Aldrich), respectively. FD-4 (400 $\left.\mu \mathrm{g} \mathrm{m} \mathrm{m}^{-1}\right)$ and HRP $(200 \mu \mathrm{g}$ $\mathrm{ml}^{-1}$ ) were added to the mucosal chamber, and samples were collected from the serosal chamber every $30 \mathrm{~min}$ for $2 \mathrm{~h}$. The concentration of FD-4 was measured via fluorescence at excitation of $485 \mathrm{~nm}$ and emission of $538 \mathrm{~nm}$. O-dianisidine substrate was used to detect HRP at absorbance $450 \mathrm{~nm}$. Samples with a fluorescent intensity values identified as outliers using Tukey's Rule were excluded from both HRP and FD4 analyses as damaged tissues. ${ }^{29}$ Data are shown as flux at 90 min.

\section{RNA extraction and quantitative RT-PCR}

Total RNA from the intestine, liver, brain and muscle tissues was extracted using TRIzol reagent (Life Technologies, Grand Island, NY) and a FastPrep-24 5G Homogenizer (MP Biomedicals, Santa Ana, CA). cDNA was synthesized from $1 \mu \mathrm{g}$ of purified RNA using iScript cDNA synthesis kit (Bio-Rad, Hercules, CA) following the manufacturer's protocol. Real-time PCR was performed with the QuantStudio 6 Flex Real-Time PCR system (Thermo Fisher Scientific, Waltham, MA) using PowerUp SYBR Green Master Mix (Thermo Fisher Scientific, Waltham, MA). GAPDH was used as a housekeeping gene. Genes of interest were analyzed according to the $2^{-\Delta \Delta \mathrm{CT}}$ method and compared with control samples. ${ }^{30}$ Primer sequences are provided in Table S2. $\dagger$

\section{Immunohistochemistry}

Hindbrains were cryosectioned at $30 \mu \mathrm{m}$ thickness and stained for c-Fos protein expression in the NTS and AP regions. Sections were permeabilized in PBST (phosphate-buffered saline containing $0.1 \%$ Tween 20 , Sigma-Aldrich), blocked in $5 \%$ normal goat serum in $0.2 \%$ Triton X-100 (Sigma-Aldrich) in PBST for $1 \mathrm{~h}$ at room temperature, and incubated overnight in a primary antibody (c-Fos at 1:100; Cell Signaling Technology, Beverly, MA) at $4{ }^{\circ} \mathrm{C}$. After washes, signals were revealed by incubation with a secondary antibody (1:500; Alexa Fluor 647, Invitrogen, Carlsbad, CA) in blocking buffer for $1 \mathrm{~h}$ in the dark at room temperature. For visualization of vagal afferents, sections were incubated with isolectin GS-IB4 Alexa Fluor 594 (IB4, 1: 500; Molecular Probes, Eugene, OR). Nuclei were counterstained with 4',6-diamidino-2-phenylindole (DAPI, 1:5000; Invitrogen) for $5 \mathrm{~min}$ followed by washes. Sections mounted on slides were closed with Prolong antifade mounting medium (Molecular Probes). Images were acquired using a confocal microscope (Leica TCS SP8 STED 3X; Leica, Wetzlar, Germany) and quantified in a blinded manner using Imaris Software (Bitplane, Zurich, Switzerland).

\section{Histology}

At the times of tissue collection, the mesenteric white adipose tissues were stored at $-80{ }^{\circ} \mathrm{C}$ until needed. For hematoxylin and eosin (H\&E) staining, a portion of the mesenteric adipose tissues were fixed in chilled $10 \%$ neutral-buffered formalin (Thermo Fisher Scientific, Waltham, MA) overnight at room temperature and transferred to $70 \%$ ethanol. Afterwards, the tissues were processed in a routine manner for paraffin sections (Tissue Tek VIP Tissue Processor; Sakura Finetek USA, Torrance, CA) and embedded the following morning. Paraffinembedded sections $(5 \mu \mathrm{m})$ were cut and stained with H\&E (Sigma-Aldrich) for microscopic examination (Olympus BX60, Waltham, MA) at $20 \times$ magnification. To quantitate adipocyte size, the H\&E-stained sections were analyzed using the ImageJ software (National Institutes of Health, Bethesda, MD). For each animal, four fields were taken from one section and the area of 10 adipocytes were measured. The images were randomized and blinded with a numerical ID.

\section{Hepatic lipid accumulation}

Hepatic lipid accumulation was qualitatively assessed by Oil Red O (ORO) staining and using hematoxylin as a counter nuclear stain. Briefly, frozen sections $(12 \mu \mathrm{m})$ were post-fixed with $4 \%$ PFA and stained with $0.37 \%$ Oil Red $\mathrm{O}$ in $60 \%$ of isopropanol for $15 \mathrm{~min}$ and washed three times with PBST. Sections were examined under a light microscope (Olympus BX60) at $40 \times$ magnification. The ORO-positive pixels were determined using ImageJ software. The hepatic triglyceride (TG) levels were measured by UCD MMPC using a commercial kit (Fisher Diagnostics, Middletown, VA). TG concentration was expressed relative to wet liver weight $\left(\mathrm{mg} \mathrm{g}^{-1}\right)$. 


\section{Microbiota DNA sequencing}

Genomic DNA from cecal samples was extracted using Zymobiomics DNA Miniprep Kit (Zymo Research, Irvine, CA). The V4 region of the 16S RNA gene was amplified in triplicate with barcoded PCR primers F515 and R806 as previously described. ${ }^{31}$ Amplicons were verified by gel electrophoresis, combined, purified, and sent to the UC Davis DNA Technologies \& Expression Analysis Core for library preparation and high throughput $250 \mathrm{bp}$ paired-end sequencing using the Illumina MiSeq platform. Resulting raw data was demultiplexed using Sabre and imported into QIIME22019.7..$^{32,33}$ Bases before base pair 21 and after base pair 242 for the forward read, before base pair 20 and after base pair 250 for reverse read were trimmed. Trimmed reads were processed with DADA2. ${ }^{34}$ Trimmed and filtered sequences were aligned and taxonomy was assigned using the 99\% SILVA naïve Bayesian classifier in QIIME 2 v2019.7. ${ }^{33}$

\section{S metagenomic analysis}

Alpha and beta diversity analyses were carried out based on the amplicon sequence variant (ASV) table rarified to 2300 reads. For determining significant differences in alpha diversity the Shannon index was calculated and differences between alpha diversity of the different treatment groups was determined using Kruskal-Wallis testing of all groups and KruskalWallis pairwise testing between groups. Beta diversity analysis was evaluated from weighted and unweighted UniFrac distance matrices. The metaMDS function in the vegan package, implemented in $\mathrm{R}$ statistical software version 4.0.0 ( $\mathrm{R}$ Core Team. 2016. R: a language and environment for statistical computing. Vienna: $\mathrm{R}$ Foundation for Statistical Computing. Available at http://www.R-project.org/), was used to generate nonmetric multidimensional (NMDS) ordination plots. $^{32}$ Pairwise PERMANOVA testing was carried out to determine if there were significant differences between diet treatment groups. To further explore key phylotypes that may contribute to the observed differences in microbial communities, microbiome taxonomic abundance data were analyzed using the linear discriminant analysis (LDA) effect size (LEfSe) method to estimate the effect size of differentially abundant features with biological consistency and statistical significance. ${ }^{35}$

\section{Metabolomic analysis}

Supernatant $(20 \mu \mathrm{l})$ from each cecum sample and standard pool dilution were reacted with $20 \mu \mathrm{l}$ of $200 \mathrm{mM} \mathrm{N}$-(3-dimethylaminopropyl)- $N$-ethylcarbodiimide hydrochloride in $5 \%$ pyridine and $40 \mu \mathrm{L}$ of $100 \mathrm{mM}$ 2-nitrophenylhydrazine in $80 \%$ $\mathrm{ACN} / \mathrm{H}_{2} \mathrm{O}(\mathrm{v} / \mathrm{v})$ with $50 \mathrm{mM} \mathrm{HCl}$. Mixtures were incubated for $30 \mathrm{~min}$ at $40{ }^{\circ} \mathrm{C}$ before adding $400 \mu \mathrm{L}$ of $10 \% \mathrm{ACN} / \mathrm{H}_{2} \mathrm{O}(\mathrm{v} / \mathrm{v})$ to each sample. Samples were centrifuged and transferred into a 96-well injection plate for triple quadrupole LC-MS/MS analysis (using Agilent 6490 triple quadrupole mass spectrometer equipped with Agilent 1290 infinity LC system, and an Agilent InfinityLab Poroshell 120 EC-C18, $2.1 \times 100 \mathrm{~mm}, 1.9 \mu \mathrm{m}$ column). The samples were detected in positive mode using a dynamic multiple reaction monitoring MRM method. ${ }^{36}$

\section{Identification of $2^{\prime}$-FL in mouse plasma}

Purification of 2 '-FL using solid phase extraction. 2'-FL was extracted from plasma essentially as described previously with the following modifications: $50 \mu \mathrm{L}$ of plasma was combined with equal volume of $50 \mathrm{mM}$ ammonium bicarbonate buffer and mixed thoroughly. ${ }^{37}$ It was then centrifuged at $4000 \mathrm{~g}$ for 30 min at $4^{\circ} \mathrm{C}$, and the supernatant was transferred to a new tube. Four volumes of a $2: 1(\mathrm{v} / \mathrm{v})$ chloroform:methanol mixture were added to this supernatant. The mixture was centrifuged at $4000 \mathrm{~g}$ for $30 \mathrm{~min}$ at $4{ }^{\circ} \mathrm{C}$ and the upper layer then was carefully transferred to a new tube. Two volumes of chilled ethanol were added, vortexed, and the mixture was left to precipitate proteins at $4{ }^{\circ} \mathrm{C}$ overnight and then centrifuged for 30 min at $4^{\circ} \mathrm{C}$. The supernatant solution containing the carbohydrates was evaporated to dryness using a centrifugal evaporator (Genevac ${ }^{\mathrm{TM}}$ miVac Centrifugal Concentrator, Ipswich, UK). The dried samples were redissolved in $150 \mu \mathrm{L}$ water and were desalted and purified via two-step solid-phase extraction (SPE) using C18 reverse-phase (Glygen ${ }^{\mathrm{TM}}$ Corp., Columbia, MD, USA) and porous graphitized carbon (PGC) (Glygen ${ }^{\mathrm{TM}}$ Corp., Columbia, MD, USA) microplates. C18 microplates were conditioned with three volumes of acetonitrile (ACN) followed by three volumes of nanopure water. The redissolved sample was loaded onto the C18 microplate and washed with three volumes of nanopure water. The flow-through was collected and subsequently loaded on a preconditioned PGC microplate. The PGC microplate was conditioned using three volumes of $80 \%$ aqueous ACN with $0.1 \%$ trifluoroacetic acid (TFA, v/v) and three volumes of nanopure water. After loading the sample onto the PGC microplate, the microplate was washed with six volumes of nanopure water to remove the salts. The desalted and purified HMOs were eluted using $40 \%$ aqueous ACN with $0.1 \%$ TFA, evaporated to dryness using Genevac ${ }^{\mathrm{TM}}$ miVac Centrifugal Concentrator (Ipswich, UK). The dried sample was redissolved in $50 \mu \mathrm{L}$ nanopure water and injected in an Agilent 6520 NanoChip LC-QToF mass spectrometer (Santa Clara, CA, USA).

Nano LC-chip-Q-TOF-MS/MS analysis. A microfluidic highperformance liquid chromatography (HPLC)-Chip that included both enrichment ( $4 \mathrm{~mm}, 40 \mathrm{~nL})$ and analytical $(75 \mu \mathrm{L}$ $\times 43 \mathrm{~mm}$ ) columns packed with $5 \mu \mathrm{m} 250 \AA$ porous graphitized carbon was used to separate the oligosaccharides. This separation was performed with binary solvent gradients of solvent $\mathrm{A}$ ( $3 \%$ ACN : $\mathrm{H}_{2} \mathrm{O}$ with $0.1 \%$ formic acid $\left.(\mathrm{v} / \mathrm{v})\right)$ and solvent $\mathrm{B}(90 \%$ ACN : $\mathrm{H}_{2} \mathrm{O}$ with $0.1 \%$ formic acid (v/v)). The gradient was set to last 45 min after the HPLC-Chip was equilibrated with 100\% solvent A and consisted of increasing from 0 to $16 \%$ B at 2.5-20 $\mathrm{min}$ and $16-100 \% \mathrm{~B}$ at $20-35 \mathrm{~min}$ followed by a $10 \mathrm{~min}$ re-equilibration of $100 \% \mathrm{~A}$.

Data was acquired in the positive ionization mode with a 300-2500 mass/charge $(\mathrm{m} / \mathrm{z})$ range. The electrospray capillary voltage was 1700-1800 V. Continuous internal calibration was performed using $\mathrm{m} / \mathrm{z} 922.009$ and 1221.991 reference masses 
(ESI-TOF Tuning Mix G1969-85000, Agilent Technologies, Santa Clara, USA). Spectra were manually examined for the presence of 2'-FL. Existence of 2'-FL and the molecular formula was confirmed using Agilent MassHunter Qualitative Analysis B.07.00 software. 2'-FL reference standard (BASF corp., Germany) and samples were prepared and analyzed in duplicate, and 2'-FL composition was confirmed by performing tandem MS. A ramped collision energy of slope 1.3 and an offset of $-3.5 \mathrm{~V}$ were used. The isolation width was medium for tandem MS and the acquisition rate was set to 1 spectra pers.

\section{Statistical analysis}

Unless stated otherwise (microbiome analysis), statistical analysis was performed by using Prism software (Prism 8.4; GraphPad Software, La Jolla, CA). Data are presented as means \pm SEMs. ROUT test was used to exclude outliers. Two-way analysis of variance (ANOVA) followed by Tukey's (when the interaction was significant) or Sidak's (for the main effects) posthoc test was used to test for differences among groups. Repeated measures ANOVA was performed on variable clusters with a random subject effect whereas variable cluster members, diet and 2'-FL treatment, were used as fixed effects. Correlations between cecal microbiome abundance and metabolites and between cecal microbiome or metabolites and metabolic parameters were determined by using the nonpara- metric Spearman and parametric Pearson correlation, respectively. Differences were considered significant if $P<0.05$. Data are presented as means \pm SEMs.

\section{Results}

\section{2'-FL supplementation improves HF-diet induced phenotype}

HF feeding significantly increased body weight compared to LF control group (LF/CON vs. HF/CON, $P<0.05$ at week $4, P<$ 0.01 at week $5 \mathrm{~s}, P<0.01$ at week $6, P<0.001$ at week $7, P<0.01$ at week 8; Fig. 1A) and significantly increased feed efficiency (LF/CON vs. HF/CON, $P<0.01$ ). However, 2'-FL supplementation had no significant effect on body weight or feed efficiency in either LF or HF fed mice (Fig. 1A and B). 2'-FL supplementation significantly decreased energy intake in HFfed mice (HF/2'-FL vs. HF/CON, $P<0.05)$ (Fig. 1C). HF feeding significantly increased fat mass in both control and 2 '-FLtreated groups (LF/CON vs. HF/CON, $P<0.0001$ at week 2, 4, 6, $P<0.001$ at week 8; LF/2'-FL vs. HF/2'-FL, $P<0.05$ at week 2, 8; Fig. 1E). 2'-FL supplementation significantly decreased fat mass in mice fed a HF diet (HF/2'-FL vs. HF/CON, $P<0.01$ at week $2,4, P<0.05$ at week 6,8$)$. There was no difference in lean mass by either diet or 2'-FL supplementation (Fig. 1D). Fasting blood glucose levels were significantly increased by $\mathrm{HF}$ feeding but there was no significant effect of 2'-FL supplemen-
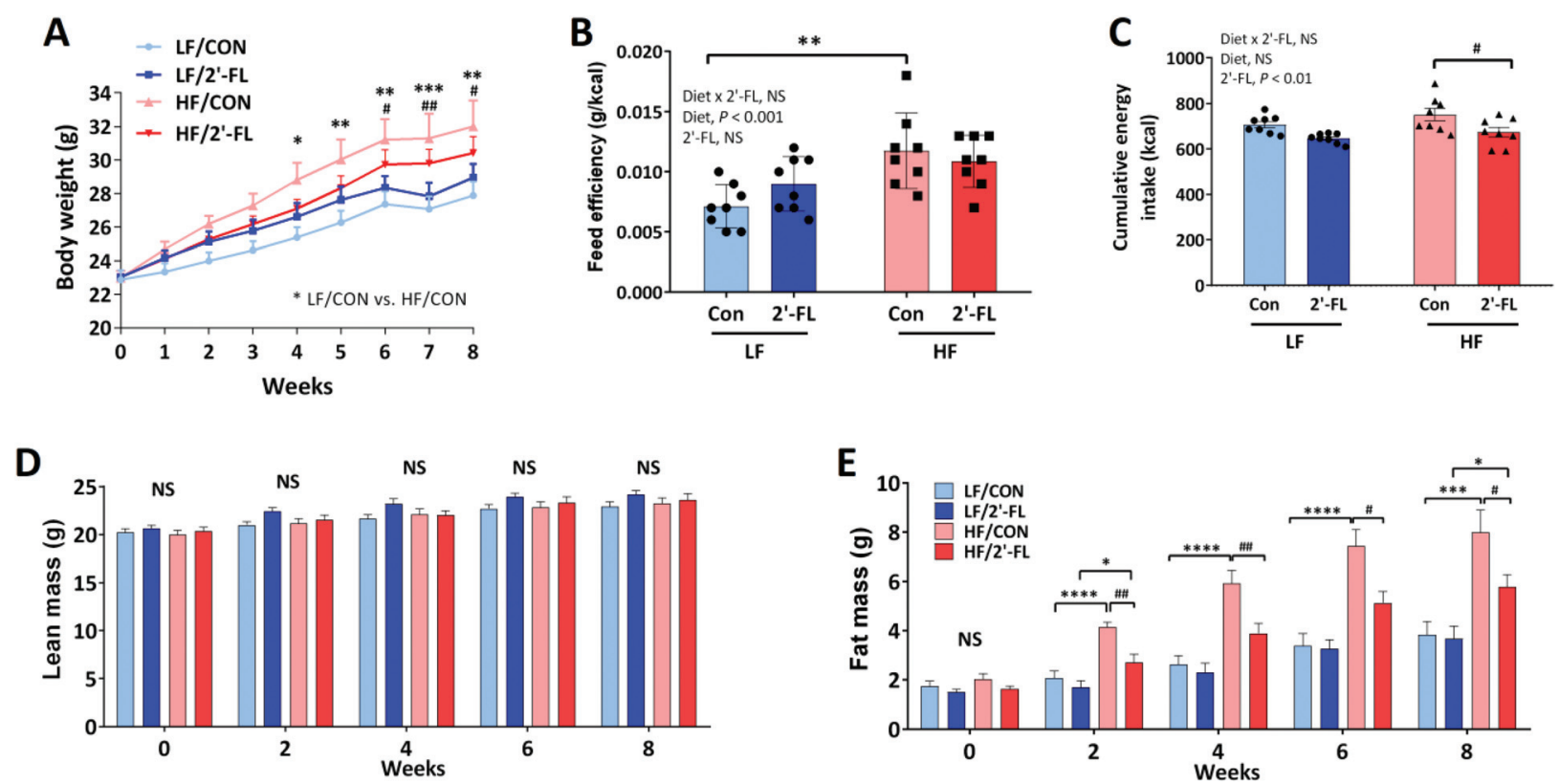

Fig. 1 Effect of 2'-FL supplementation on metabolic phenotype. Body weight (A), feed efficiency (weight gain (g)/energy intake (kcal); B), cumulative energy intake (C), and lean and fat mass (D and E) in mice fed an LF or HF with or without $10 \% 2$ '-FL supplementation diet for 8 weeks. Two-way analysis of variance (ANOVA) followed by Tukey's or Sidak's post-hoc test was used to determine differences among groups. Repeated measures ANOVA was performed for body weight and body composition on variable clusters with a random subject effect whereas variable cluster members, diet and 2'-FL treatment, were used as fixed effects. Values are means \pm SEMs; $n=8 /$ group. A: Asterisk $\left({ }^{*}\right)$ or sharp $\left({ }^{\#}\right)$ denote significant differences between LF/Con vs. HF/Con or LF/2'-FL vs. HF/2'-FL; B-E: asterisk $\left(^{*}\right)$ or sharp $\left(^{\#}\right)$ denotes significant differences between LF vs. HF or Con vs. $2^{\prime}-\mathrm{FL}$

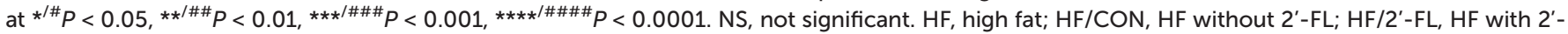
$\mathrm{FL}(\mathrm{w} / \mathrm{w})$ in diet; $\mathrm{LF}$, low fat; LF/CON, LF without 2'-FL; LF/2'-FL, LF with 2'-FL (w/w) in diet; 2'-FL, 2-fucosyllactose. 
tation on this increase or on the impaired response to an oral glucose load (Fig. S1†).

Lipid metabolism was examined in the liver and adipose tissue (Fig. S2 and S3†). There were no significant effects by diet or 2'-FL supplementation on hepatic lipid accumulation or triglyceride (Fig. S2A-C $\dagger$ ). However, 2'-FL supplementation significantly suppressed the HF-feeding induced increase in gene expression of peroxisome proliferator-activated receptor gamma (PPAR $\gamma$ ), a transcription factor for adipogenesis (LF/ $\mathrm{CON}$ vs. $\mathrm{HF} / \mathrm{CON}, P<0.01 ; \mathrm{HF} / \mathrm{CON}$ vs. $\mathrm{HF} / 2^{\prime} \mathrm{FL}, P=0.001$; Fig. S2D $\dagger$ ). In addition, 2 '-FL supplementation significantly downregulated the gene expression of SREBP-1c, a transcription factor involved in lipogenesis, in a HF fed mice (LF/2'-FL vs. HF/ $2 '$-FL, $P<0.001$; Fig. S2E $\dagger$ ). In visceral adipose tissues, 2 '-FL supplementation significantly decreased the size of adipocytes compared to control groups in mice fed either a LF or a HF diet (LF/ CON vs. LF/2'-FL, $P<0.01 ; \mathrm{HF} / \mathrm{CON}$ vs. HF/2'-FL, $P<0.05$; Fig. S3 $\dagger$ ). There was, however, no change in expression of other markers for lipid metabolism in the liver and visceral adipose tissues (Fig. S3†). There were no differences in gene expression in either fast-twitch (gastrocnemius and tibialis anterior) or slow-twitch (soleus) muscles (Table S3†).

\section{2'-FL supplementation preserves the intestinal barrier function}

Intestinal permeability in the cecum was evaluated ex vivo using an Ussing chamber system (Fig. 2A and B). There was no significant difference in either paracellular or transcellular permeability between LF and HF fed mice. However, there was a significant treatment effect of 2'-FL supplementation to decrease paracellular and transcellular permeability in both LF and HF fed mice (Fig. 2B). There was no change in cecal gene expression of markers for intestinal barrier function and tight junction function (Table S4 $\dagger$ ). However, expression of Reg3 $\gamma$ was significantly increased by 2 -FL supplementation in the ileum of LF fed mice. In addition, 2 '-FL supplementation significantly increased IL-22 gene expression, a key cytokine regu- lating epithelial homeostasis, in the ileum of both $\mathrm{LF}$ and $\mathrm{HF}$ fed mice (LF or HF/CON vs. LF or HF/2'-FL, $P<0.05$; Fig. 2C).

\section{2'-FL supplementation restores the integrity of vagally- mediated gut-brain signaling}

To investigate the ability of CCK to activate second order neurons in the brainstem, CCK was administered 2 hours before euthanasia and hindbrain tissues were immunostained to determine c-Fos expression, a marker for neuronal activation in the AP and NTS regions of the brainstem where vagal afferents terminate (Fig. 3A-C). 2'-FL-treated mice on a HF diet showed higher number of c-Fos-positive cells in both AP (HF/ $\mathrm{CON} v$ s. HF/2'-FL, $P=0.085$; Fig. 5B) and NTS (HF/CON vs. HF/ 2 '-FL, $P<0.05$; Fig. 5C) areas compared to the control mice on a HF diet. There was no significant difference between LF-fed groups in the number of c-Fos-positive cells in both areas.

\section{Effect of 2'-FL supplementation on cognitive functions}

Cognitive functions in mice were determined by Y-maze (Fig. 4A and B), open-field (Fig. 4C and D) and novel object recognition (Fig. 4E) tests. For spatial learning and memory evaluated by the Y-maze test, no differences were found with diet (LF or HF) or treatment (CON or 2'-FL) and there were no interactions between diet and treatment for the number of arm entries (Fig. 4A) and spontaneous alternation (Fig. 4B). For locomotor activity and anxiety-related behavior determined by the open-field test, there was a trend for diet to influence duration (s) in center vs. perimeter (Fig. 4C and D). The control mice tended to spend more time in the center compared to HF-fed mice ( $p=0.083)$. This difference was driven by a trend for a diet $\times$ treatment interaction where $\mathrm{HF} / 2$ '-FL animals spent more time in the perimeter $(p=0.082)$. For short-term non-associative memory evaluated by the novelobject test, no differences $(p>0.1)$ were found with diet (LF or $\mathrm{HF})$ or treatment (CON or 2 '-FL) and there were no interactions
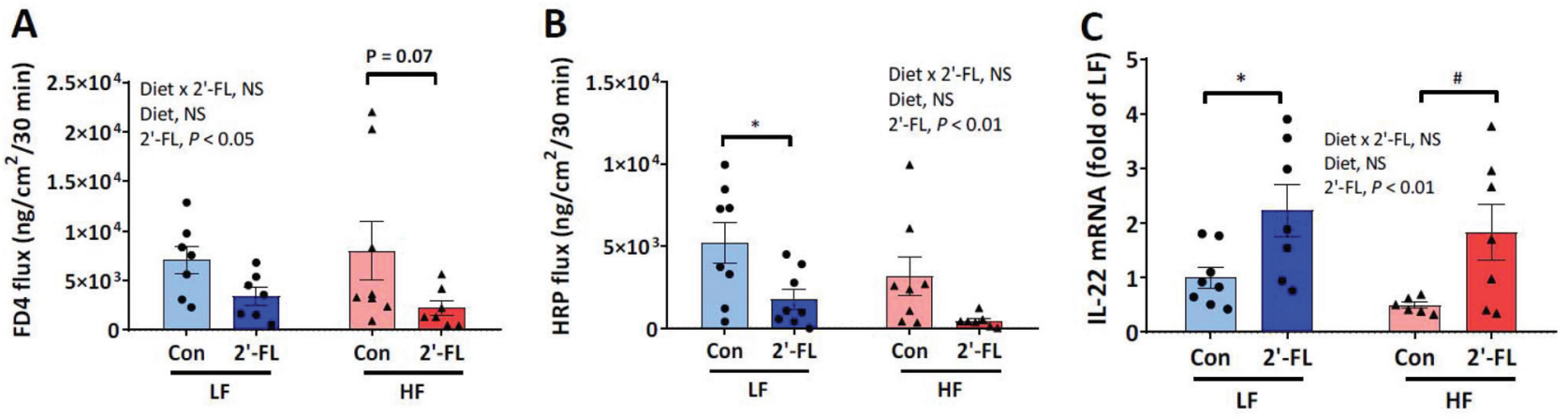

Fig. 2 Effect of 2'-FL supplementation on gut barrier integrity. FD4 flux (paracellular pathway; A), HRP flux (transcellular pathway; B) in the cecum and IL-22 gene expression in the ileum (C) of mice fed an LF or HF with or without 10\% 2'-FL supplementation diet for 8 weeks. Two-way analysis of variance followed by Tukey's or Sidak's post-hoc test was used to determine differences among groups. Values are means \pm SEMs; $n=8 / g r o u p$. Asterisk $(*)$ or sharp $(\#)$ denotes significant differences between LF vs. HF or Con vs. $2^{\prime}-\mathrm{FL}$ respectively at $P<0.05$. NS, not significant. FD4, fluorescein isothiocyanate-dextran 4000; HF, high fat; HF/CON, HF without 2'-FL; HF/2'-FL, HF with 2'-FL (w/w) in diet; HRP, horseradish peroxidase; LF, low fat; LF/CON, LF without 2'-FL; LF/2'-FL, LF with 2'-FL (w/w) in diet; 2'-FL, 2-fucosyllactose. 
A
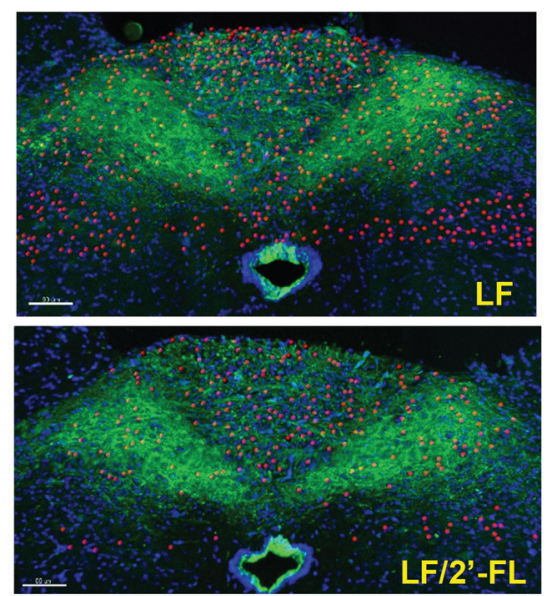

Scale bar: 70 um

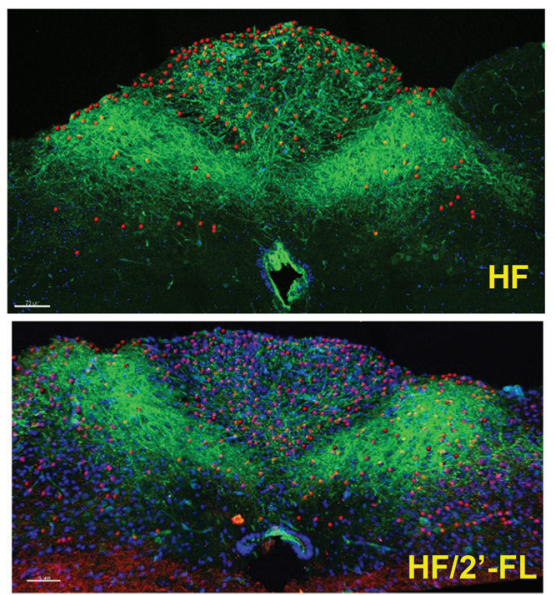

HF

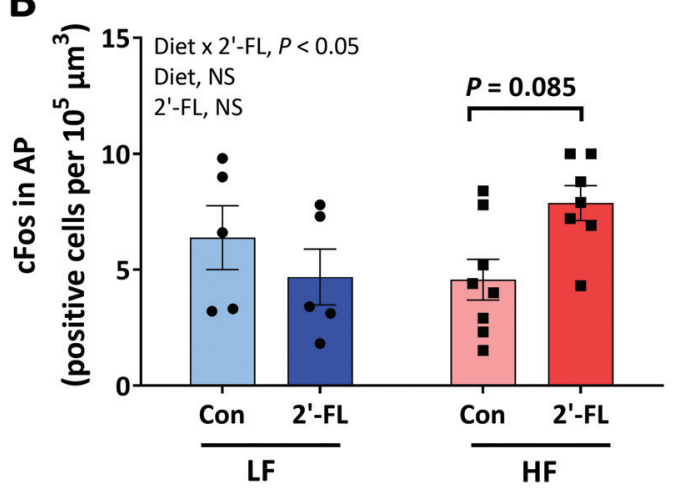

C

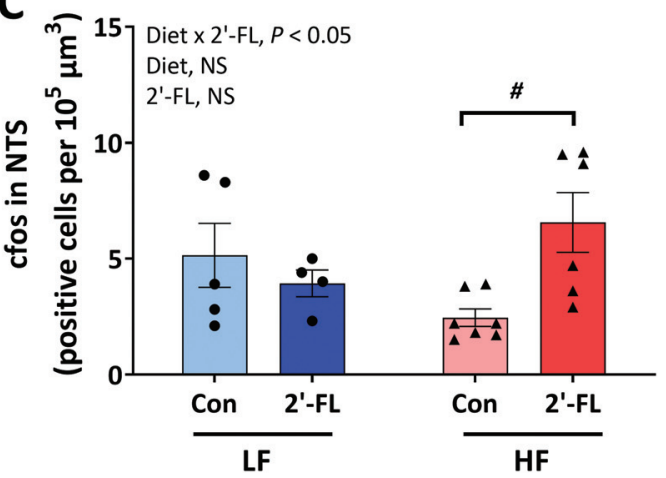

Fig. 3 Effect of 2'-FL supplementation on CCK-induced vagally-mediated gut-brain signaling. C-fos positive cells in the area postrema (AP, B) and nucleus of the solitary tract (NTS, C) of the hindbrain (A) in mice fed an LF or HF with or without $10 \% 2^{\prime}$-FL supplementation diet for 8 weeks. Red immunofluorescence represents fos-immunopositive cells, DAPI-positive nucleii are depicted in purple and IB4 immunofluorescence depicted in green. Two-way analysis of variance followed by Tukey's or Sidak's post-hoc test was used to determine differences among groups. Values are means $\pm \mathrm{SEMs} ; n=8$ /group. Asterisk $\left({ }^{\#}\right)$ denotes significant differences between Con vs. 2'-FL at $P<0.05$. HF, high fat; $\mathrm{HF} / \mathrm{CON}$, HF without 2'-FL; HF/2'-FL, HF with 2'-FL (w/w) in diet; LF, low fat; LF/CON, LF without 2'-FL; LF/2'-FL, LF with 2'-FL (w/w) in diet; 2'-FL, 2-fucosyllactose.

between diet and treatment for discrimination between the novel and familiar objects (Fig. 4E).

Compositional changes in the gut microbiota and metabolites driven by 2 '-FL supplementation

There were no significant differences in alpha diversity of the gut microbiota (measured by the Shannon index; Fig. 5A) among groups after 8-week of 2'-FL supplementation. However, the analysis of beta diversity using unweighted UniFrac distances showed that mice supplemented with 2 '-FL had a statistically significant difference in beta diversity compared to mice given $\mathrm{LF}$ or $\mathrm{HF}$ diets alone (LF/CON vs. LF/2'-FL, $P=$ $0.001 ; \mathrm{HF} / \mathrm{CON}$ vs. HF/2'-FL, $P=0.001$; Fig. 5B). Further, the NMDS plot of unweighted Unifrac distances also showed a clustering of microbial communities according to 2'-FL supplementation but not diet (Fig. 5C).

To further explore primary phylotypes that may contribute to the observed differences in microbial communities, the linear discriminant analysis (LDA) effect size (LEfSe) algorithm was performed (Fig. 5D). HF feeding alone altered the gut microbiota composition. Specifically, HF feeding alone led to differential increases in the relative abundance from the Firmicutes phylum of the family Lachnospiraceae (specifically Blautia and Acetatifactor) and from the Proteobacteria phylum (Parasutterella). 2'-FL supplementation differentially increased the relative abundance of Coriobacteriaceae UCG-002, Bacteroides (especially Bacteroides thetaiotaomicron species), Lactobacillus, Lachnoclostridium, Lachnospiraceae FCS020 group, and Oscillibacter.

2'-FL supplementation led to significant shifts in cecal metabolites (Fig. 6; Fig. S8 †). In particular, concentrations of glyceric and lactic acids were significantly elevated by 2 '-FL supplementation compared to the LF/CON and HF/CON group, respectively (LF/CON vs. LF/2'-FL, $P<0.05 ; \mathrm{HF} / \mathrm{CON} v s$. $\left.\mathrm{HF} / 2^{\prime}-\mathrm{FL}, P<0.05\right)$. On the other hand, the concentration of indole-3-acetic acid was significantly reduced by 2 '-FL supplementation in both LF and HF-fed groups (LF/CON vs. LF/2'FL, $P<0.01 ; \mathrm{HF} / \mathrm{CON} v s$. HF/2'-FL, $P<0.001)$. 
A
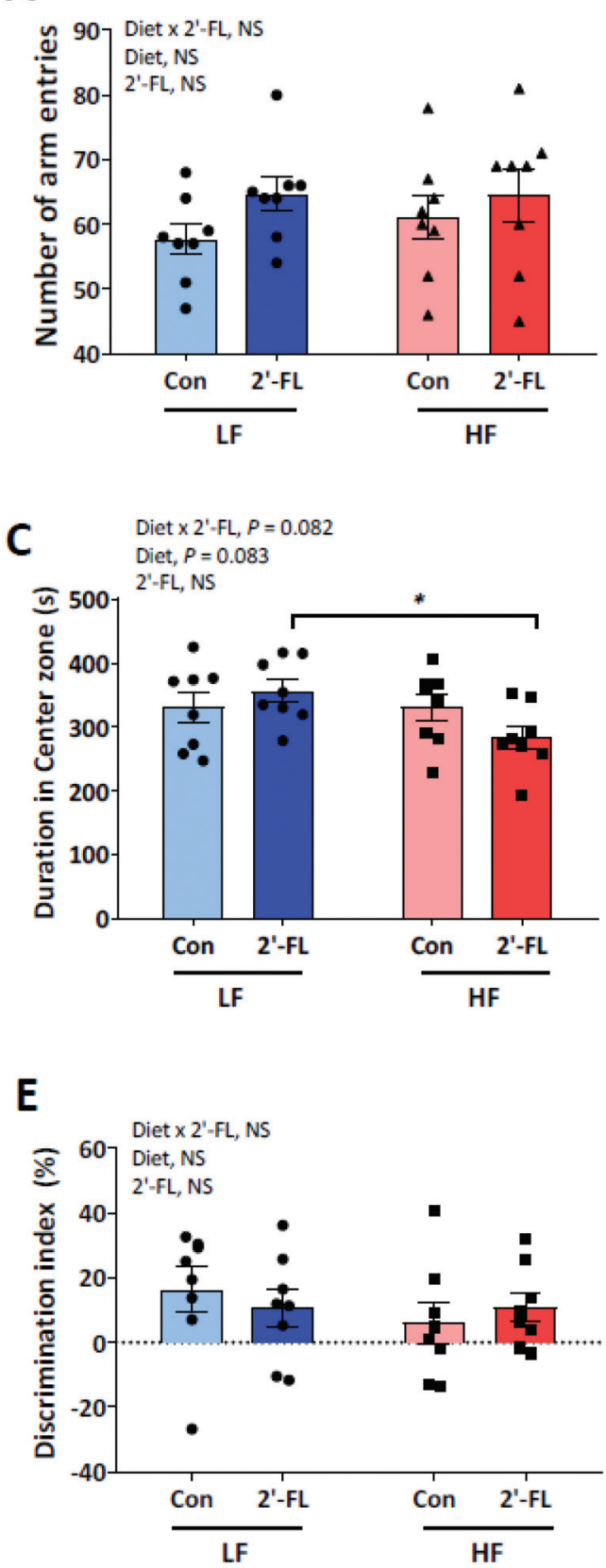

B
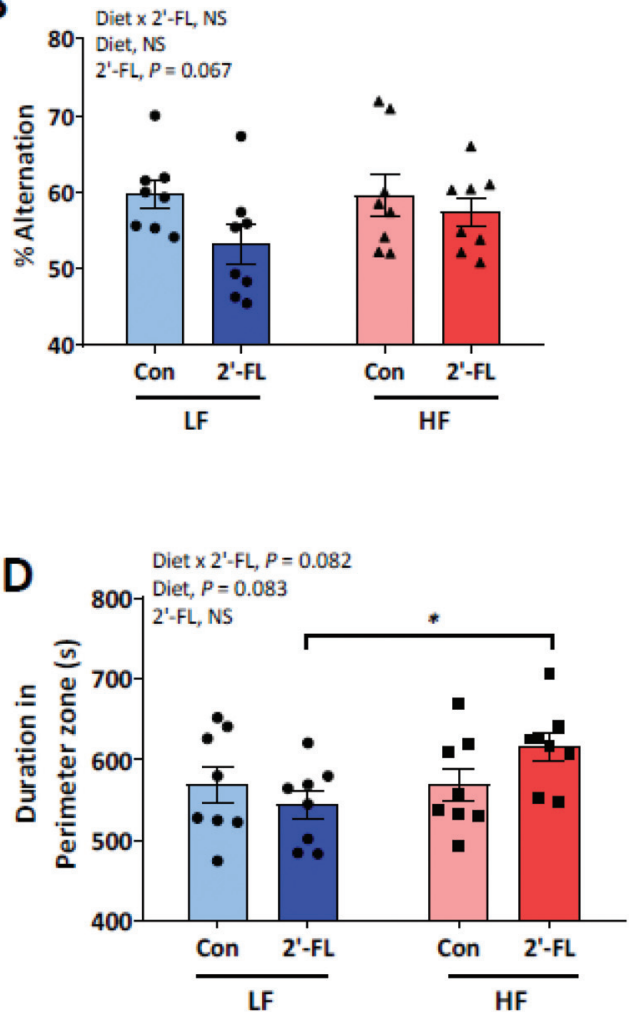

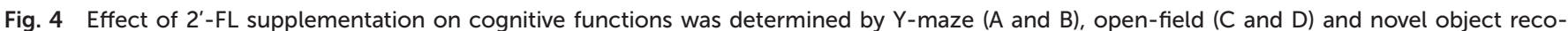

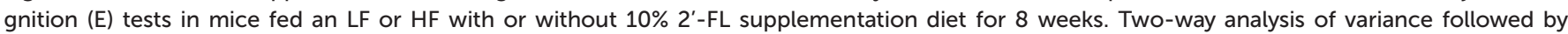

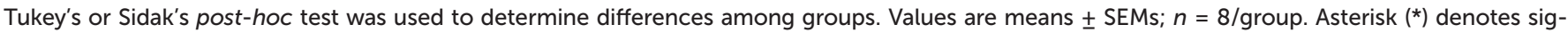

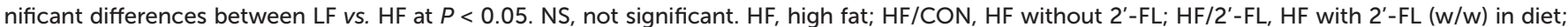
LF, low fat; LF/CON, LF without 2'-FL; LF/2'-FL, LF with 2'-FL (w/w) in diet; 2'-FL, fucosyllactose.

Correlation analysis showed that there were two distinct clusters based on the strong correlation between bacterial taxa and metabolites (Fig. 7A). Cluster 1, including Lachnospiraceae NK4A136 group, Blautia, Acetatifactor, and Parasutterella, was positively correlated with indole-3-acetic acid and negative correlated with glyceric acid. In contrast, cluster 2, including Coriobacteriaceae UCG-002 Bacteroides (thetaiotaomicron),
Lactobacillus, Lachnoclostridium, and Lachnospiraceae FCS020 group, showed a positive correlation with glyceric acid and a negative correlation with indole-3-acetic acid. These two clusters were also correlated with the major metabolic parameters of this study related to the lipid metabolism (ORO, PPAR $\gamma$, SREPB-1c), and gut barrier integrity (FD4, HRP, IL-22) (Fig. 7B). Specifically, cluster 1 was positively correlated with 
A

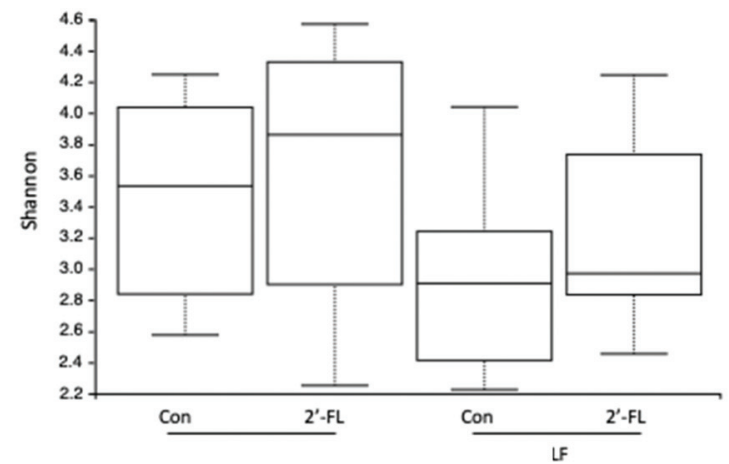

B

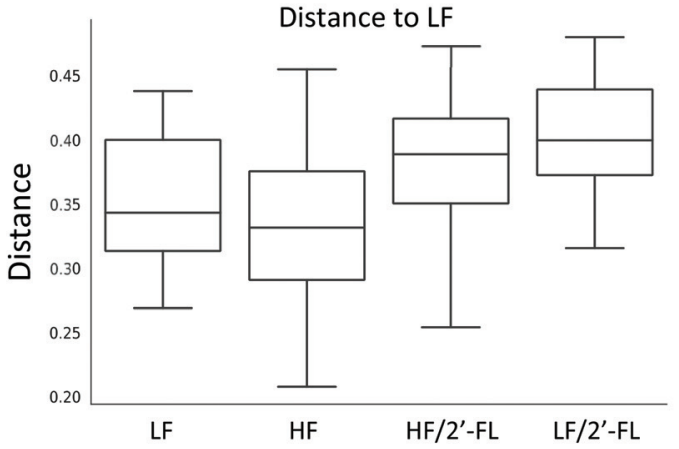

C

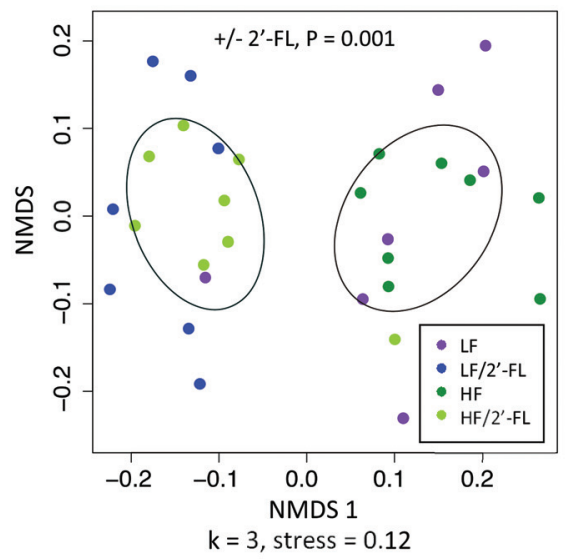

D
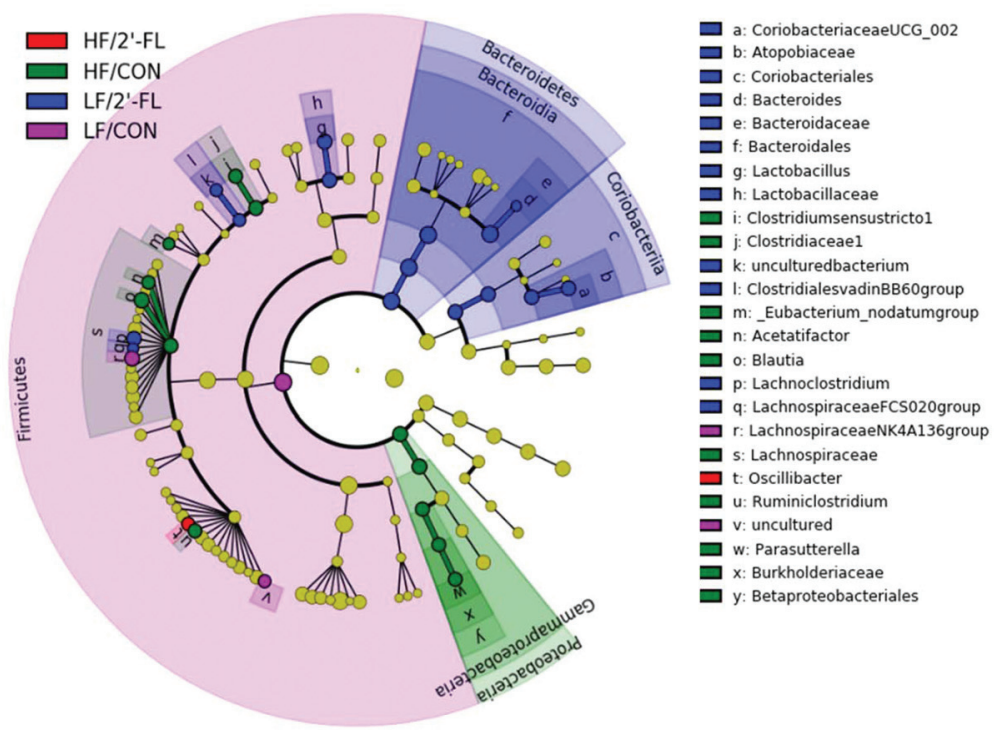

Fig. 5 Effect of $10 \% 2^{\prime}-$ FL supplementation on the composition of the gut microbiota. Alpha diversity box plot showing Shannon richness of the microbial communities separated by diet (A), multigroup comparison box plot (beta diversity index) of unweighted UniFrac distance (B), unweighted UniFrac NMDS with points colored by diet, ellipses are drawn based on the standard deviation of the points with respect to the addition or absence of $2^{\prime}-\mathrm{FL}$ in the diet (C) of mice fed an LF or HF with or without $10 \% 2^{\prime}-\mathrm{FL}$ supplementation diet for 8 weeks. Differences among alpha diversity of the different treatment groups was calculated using Kruskal-Wallis testing of all groups. PERMANOVA testing of unweighted UniFrac distances was used to determine differences among groups for beta diversities. The linear discriminant analysis (LDA) effect size (LEfSe) method was used to identify taxa that were significantly differentially abundant for each group. $n=7-8 /$ group. Cladogram (D) generated from LEfSe analysis shows the most differentially abundant taxa enriched in microbiota; purple indicating LF/CON, blue LF/2'-FL, green HF/CON, red HF/2'-FL, and yellow indicating non-significance. The six rings of the cladogram stand for domain (innermost), phylum, class, order, family and genus. HF, high fat; HF/CON, HF without 2'-FL; HF/2'-FL, HF with 2'-FL (w/w) in diet; $L F$, low fat; $L F / C O N$, LF without 2'-FL; LF/2'-FL, LF with 2'-FL (w/w) in diet; 2'-FL, 2-fucosyllactose.

fat accumulation (ORO, PPAR $\gamma$, SREPB-1c) and increased gut permeability (FD4, HRP) and negatively correlated with c-Fos expression in the NTS and gut barrier integrity (IL-22). On the other hand, cluster 2 was positively correlated with c-Fos expression in the NTS and gut barrier integrity (IL-22) and negatively correlated with fat accumulation (ORO, PPAR $\gamma$, SREPB-1c) and increased gut permeability (FD4, HRP). Further, these major metabolic biomarkers also showed the same trend of correlation with the concentrations of the metabolites that are positively correlated with the two clusters of bacterial taxa (Fig. 7B). That is, indole-3-acetic acid or glyceric acid was positively or negatively correlated with fat accumulation and increased gut permeability while negatively or posi- tively correlated with c-Fos expression in the NTS and gut barrier integrity.

\section{Identification of 2'FL in mouse plasma using Nano-LC chip QTOF MS/MS}

2'-FL was identified using a nano-LC chip QTOF, and tandem MS was used to confirm identity. A representative tandem MS spectrum of 2'-FL, showing its typical oligosaccharide fragmentation patterns with b- and y-type ions, is depicted in Fig. S9. $\dagger$ The MS/MS spectra of protonated 2'-FL (precursor ion at $\mathrm{m} / \mathrm{z}$ 471.18 derived from ion $489.18 \mathrm{~m} / \mathrm{z}$ minus one molecule of water) displays the consecutive loss on fucose and a terminal hexose residue yielding ions at $m / z 325.11$ and 309.12, respect- 

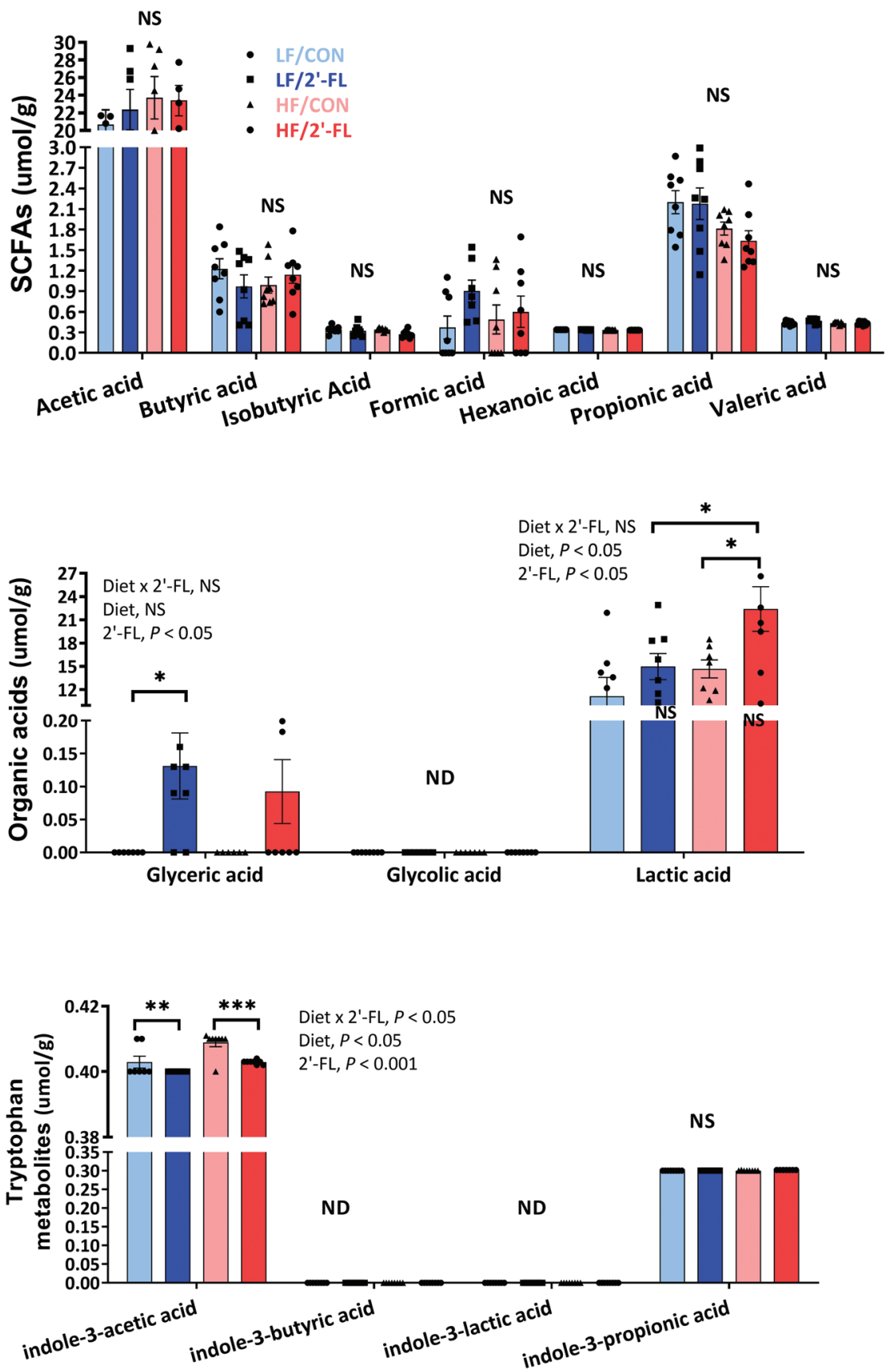

Fig. 6 Effect of 10\% 2'-FL supplementation on metabolomic profiles in the cecum of mice fed an LF or HF with or without $10 \%$ 2'-FL supplementation diet for 8 weeks. Two-way analysis of variance (ANOVA) followed by Tukey's or Sidak's post-hoc test was used to determine differences among groups. Values are means \pm SEMs; $n=8$ /group. Asterisk $\left(^{*}\right)$ or sharp $\left({ }^{\#}\right)$ denotes significant differences between LF vs. HF or Con vs. $2^{\prime}-\mathrm{FL}$

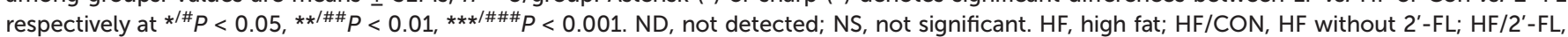
HF with 2'-FL (w/w) in diet; LF, low fat; LF/CON, LF without 2'-FL; LF/2'-FL, LF with 2'-FL (w/w) in diet; SCFA, short chain fatty acid; $2^{\prime}-F L$, 2-fucosyllactose.

ively. These characteristic fragments were missing from the spectra of the plasma samples collected from mice that had not received 2'-FL supplementation. A summary of the presence or absence of $2^{\prime}$-FL in plasma of mice fed a low or highfat diet with or without $2^{\prime}$ FL supplementation is shown in ESI Table 6. $\dagger$

\section{Discussion}

In the present study, we investigated the effects of 2 '-FL supplementation on metabolic and cognitive functions and on the gut microbiota in mice with or without the metabolic challenge of HF feeding. Our hypothesis was that 2'-FL supplemen- 

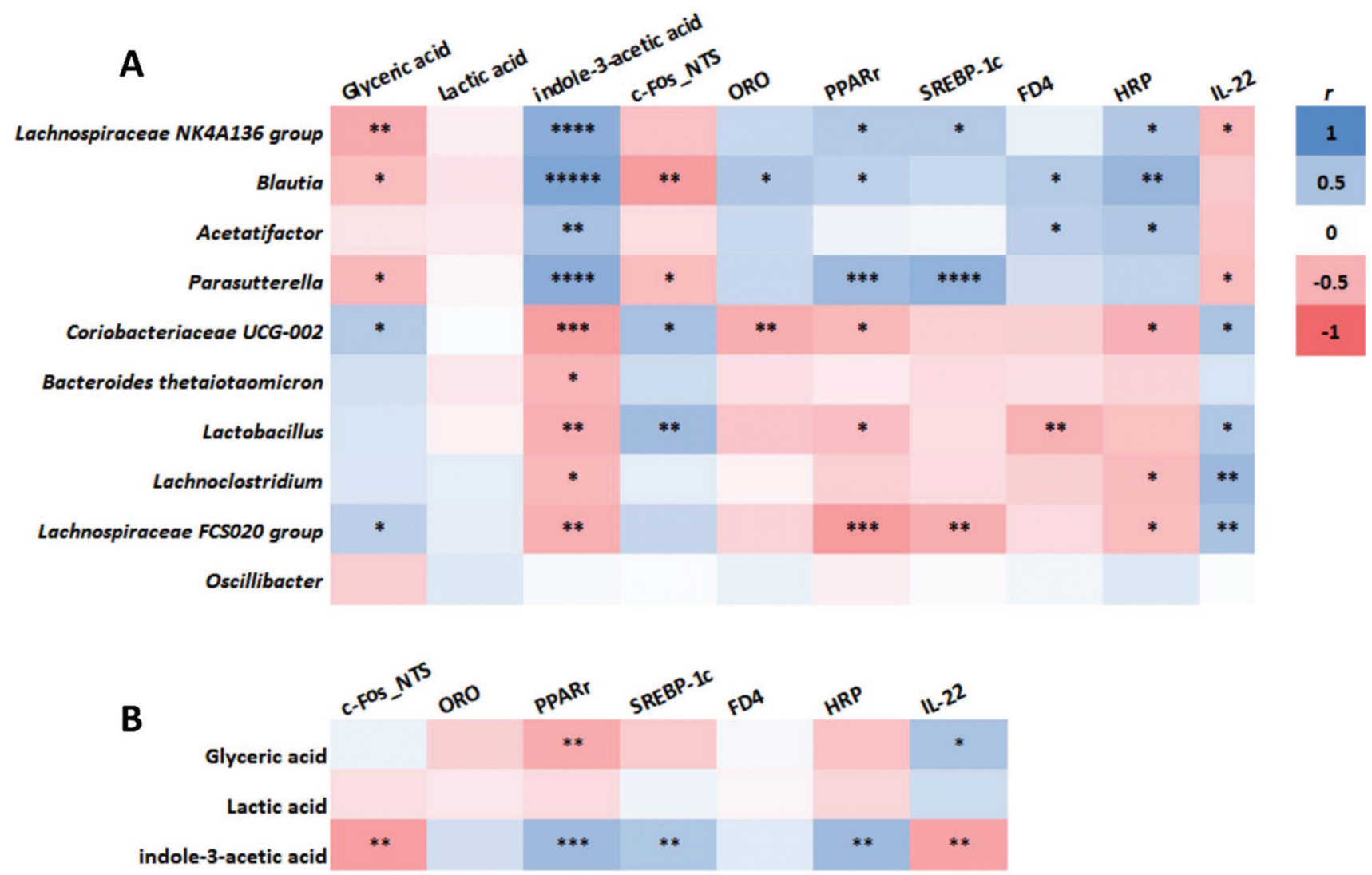

Fig. 7 Correlation of the cecal microbiota of significant differences, microbial metabolites, and metabolic parameters in mice fed an LF or HF with or without $10 \% 2$ '-FL supplementation diet for 8 weeks. Correlation of the gut microbiota with metabolites and metabolic biomarkers (A) and correlation between metabolites and metabolic parameters (B) were determined by the nonparametric Spearman and parametric Pearson correlation analysis, respectively. $n=7-8$ /group. Asterisk $\left(^{*}\right)$ denotes significant differences among groups at $* P<0.05,{ }^{* *} P<0.01,{ }^{* * *} P<0.001,{ }^{* * * *} P<0.0001$, $\star \star \star \star P<0.000001$. HF, high fat; HF/CON, HF without 2'-FL; HF/2'-FL, HF with 2'-FL (w/w) in diet; LF, low fat; LF/CON, LF without 2'-FL; LF/2'-FL, LF with 2'-FL (w/w) in diet; 2'-FL, 2-fucosyllactose.

tation would change the gut microbiota composition and this would be associated with improvements in metabolic and cognitive profiles. 2'-FL supplementation led to compositional shifts in the gut microbiota which was characterized by a differential increase in the relative abundance of the Bacteroides genus, in particular Bacteroides thetaiotaomicron species, which has been shown to contribute to gut barrier integrity. ${ }^{38} 2^{\prime}$-FL supplementation also increased the level of cecal glyceric acid consistent with our previous findings. ${ }^{7}$ The compositional changes in the gut microbiota were associated with improvements in gut barrier integrity and signaling in the vagal afferent pathway, which were compromised by the HF-diet. 2'-FL supplementation also improved a number of major metabolic parameters including body weight and adiposity, and lipid metabolism, as previously reported. ${ }^{7}$ However, there was no detectable effect on cognitive function. Taken together, these data show that 2'-FL supplementation led to improvements in gut permeability, signaling in the vagal afferent pathway and the metabolic profile in HF-fed mice. These improvements were associated with changes in the composition of the gut microbiota and its metabolites.
A primary mechanism for the beneficial effects of prebiotics is, by definition, via changes in the gut microbiota. In the present study, diet alone had little effect on the overall composition of the gut microbiota composition at all taxonomic levels; HF and LF-fed groups showed similar profile of the gut microbiota. We did not observe the diet-induced dysbiotic changes as previously characterized in HF-fed animals such as an increased ratio of Firmicutes to Bacteroidetes relative abundance. ${ }^{39}$ This is likely due to the similar composition of the semi-purified diets used in the present study in which the fiber content of the diet was carefully controlled. ${ }^{40}$ However, we did observe a differential increased the relative abundance of the Blautia genus in mice fed a HF diet consistent with our previous study. ${ }^{7}$ In contrast, $2^{\prime}$-FL supplementation in mice fed either the LF or HF diet led to distinct and significant changes in the gut microbiota profile compared to the control groups, suggesting that 2 'FL has a significant effect on the composition of the gut microbiota. The gut microbiota composition of the 2'-FL-fed group was characterized by differential increase in the relative abundance of the Bacteroides genus, which was primarily driven by the Bacteroides thetaiotaomicron 
species. Colonization of germ-free mice with this species has previously been shown to modulate the expression of genes involved in important intestinal functions including increased gut barrier integrity, similar to the effects of 2 '-FL seen in the present study. ${ }^{38}$ Our findings are consistent with published data showing that 2'-FL treatment increases the abundance of the Bacteroides genus in culture and that the HMOs 3'-sialyllactose $\left(3^{\prime}-\mathrm{SL}\right)$ and $6^{\prime}$-SL increase the growth of Bacteroides thetaiotaomicron. ${ }^{41}$

We characterized the microbial metabolites, specifically short chain fatty acids and indole derivatives, in the cecal contents as another measure of the effect of $2^{\prime}$-FL supplementation on the gut microbiota. ${ }^{42}$ Overall, there was little effect of diet on the microbial metabolites consistent with the lack of overall effect of diet on the composition of the gut microbiota. However, 2'-FL supplementation significantly increased glyceric acid and lactic acid and significantly decreased indole-3acetic acid. We found two distinct clusters of microbial taxa based on the correlation between bacterial taxa and metabolites. Cluster 1, including Lachnospiraceae NK4A136 group, Blautia, Acetatifactor, and Parasutterella whose relative abundances were differentially increased by HF feeding, was positively correlated with indole-3-acetic acid and negatively correlated with glyceric acid. Conversely, cluster 2, including Coriobacteriaceae UCG-002, Bacteroides (thetaiotaomicron), Lactobacillus, Lachnoclostridium, and Lachnospiraceae FCSO2O group whose relative abundances were differentially increased by 2 '-FL supplementation showed a positive correlation with glyceric acid and a negative correlation with indole3 -acetic acid. Further, these two clusters and the positively-correlated metabolites were also correlated with the beneficial effect on the major metabolic parameters shown in the present study. The significance of an increase in lactic and glyceric acids in mediating the beneficial effects of $2^{\prime}$-FL is not tested here. Lactate has been shown to act through the G-protein coupled receptor Gpr81. Activation of Gpr81 in mice colonized with a lactate producing Lactobacillus species stimulated colonic stem cell proliferation. ${ }^{43}$ Lactate is also protective in rodent models of colitis ${ }^{44}$ and it is possible that this plays a part in the improvement of intestinal barrier function seen in the present study. Little is known about the potential effects of bacterially produced glyceric acid in the host.

Microbiota and their metabolites are in close proximity to the gut epithelium and can play a key role in alteration of intestinal barrier function. ${ }^{45} \mathrm{HF}$ feeding is associated with an increase in intestinal permeability and there is evidence that this can lead to systemic inflammation and also altered neuronal function, including in the central nervous system and in the vagal afferent pathway. ${ }^{4,46}$ We hypothesized that the 2 '-FLinduced compositional changes in gut microbiota and metabolites would be associated with improved gut barrier integrity. We found that cecal permeability was decreased by 2 '-FL supplementation. However, this improved intestinal barrier integrity in the cecum was not associated with changes in gene expression of proteins associated with tight junction and epithelial function. In the ileum, there was an increase in gene expression of IL-22, a member of the IL-10-related family of cytokines and known to be critical for gut health as a key cytokine regulating epithelial homeostasis. ${ }^{47}$ These intestinal protective effects of 2'-FL were positively correlated with 2'-FL-driven microbiota taxa and metabolites. IL-22 expression is downstream of the aryl-hydrocarbon receptor (AhR), which has been shown to be activated by tryptophan derivatives, including indole-3-acetic acid. We found indole-3-acetic acid to be decreased in the cecum of 2'FL supplemented mice and thus it is unlikely that AhR plays a major role in the beneficial effects of 2'-FL. There are other potential mechanisms; genera from family Lachnospiraceae have been associated with the maintenance of gut health. ${ }^{48}$ Lachnospiraceae FCSO2O is a member of a common butyrate-producing gut bacterial taxa associated with diets high in fiber and complex carbohydrates and a recent study also reported a significant increase in Lachnospiraceae FCS020 group in mice supplemented with polysaccharides, which was associated with improved metabolic profiles including systemic inflammation. ${ }^{49,50}$ However, no change in cecal concentrations of butyrate with $2^{\prime}$-FL treatment was detected in the current study.

Impaired intestinal barrier function has been associated with obesity, systemic inflammation and altered neuronal function. We found little effect of either diet or 2'-FL supplementation on plasma lipopolysaccharide (LPS)-binding protein (LBP) level (a proxy measure of LPS in the circulation), albeit the highest level of LBP was found in HF fed mice consistent with other reports. Dysregulation of the microbiota-gutbrain axis is considered as the potential mechanism by which a chronic HF diet induces neuroinflammation and cognitive impairment. ${ }^{9,51}$ Recent studies have demonstrated that dietary supplementation with prebiotics can prevent cognitive impairment via the microbiota-gut-brain axis. ${ }^{8-11}$ In the present study, 2 '-FL supplementation had no measurable effect on cognitive function. In the majority of studies investigating the effect of $\mathrm{HF}$ diets on cognitive function, $\mathrm{HF}$ diets were provided for more than 3-month period, allowing sufficient duration to induce cognitive deficits or mature adult mice (3-6 month old) were used for age-susceptibility to cognitive decline. ${ }^{8-10,52}$ A significant limitation of the current study is that HF feeding itself had no detrimental effect on cognitive performance, possibly due to the duration of treatment and the age of the mice. Thus, the use of longer feeding duration and aged mice with larger sample size might better enable the inducement of cognitive impairment and further validate the possible beneficial effects of 2'-FL supplementation on cognitive profiles.

Supplementation with 2'-FL improved vagal afferent signaling, as shown by an increase in CCK-induced c-Fos immunoreactivity in the brainstem. This is consistent with the $2^{\prime}$-FLinduced decrease in overall food intake in HF-fed mice as improved intestinal satiety signaling via the vagal afferent pathway would be predicted to decrease food intake. Whether this improved vagal afferent signaling is due to changes in gut microbial metabolites, improved intestinal permeability or a direct effect of 2 '-FL requires additional studies.

HF feeding leads to accumulation of adipose tissue with hypertrophic adipocytes and abnormal hepatic lipid metab- 
olism, which is associated with increased expression of hepatic PPAR $\gamma$ and SREBP-1c as transcription factors essential for adipocyte differentiation and biosynthesis of fatty acids and triglycerides. ${ }^{53,54}$ In the present study, HF-induced increases in fat mass and adipocyte hypertrophy were reduced by 2 '-FL supplementation. It is interesting to note that 2 '-FL supplementation also significantly reduced the adipocyte size in LF-fed mice, demonstrating the ability of 2'-FL to improve lipid metabolism in adipose tissue even without HF-driven metabolic challenges possibly via a decrease in adipogenesis and lipogenesis and/or an increase in lipolysis. ${ }^{55}$ In line with improved lipid metabolism in adipose tissue, 2'-FL supplementation also significantly downregulated the overexpression of PPAR $\gamma$ and SREBP-1c genes by HF feeding in the liver. There is accumulating evidence that compositional and functional dysbiosis of the gut microbiota may play a crucial role in the development of abnormal hepatic lipid metabolism. Notably, the gene expression of PPAR $\gamma$ and SREBP-1c were strongly negatively correlated with the HF-driven Parasutterella genus in this study, whose increased abundance has been observed in obesity-related conditions including increases in adipogenesis. ${ }^{56}$ Thus, it is suggested that the normalization of the HF-induced dysbiosis by 2 '-FL supplementation may have contributed to improvement in lipid profiles in the liver. It is possible that 2 '-FL can be absorbed across the gut epithelium, enter the bloodstream and exert systemic effects. The presence of intact HMOs, including 2'-FL, has been previously reported in samples of urine, blood and serum. ${ }^{37,57-59}$ Here we show the presence of intact $2^{\prime}$-FL in the plasma of both LF and HFfed mice with $2^{\prime}$ FL supplementation, demonstrating its absorption from the gut and its potential to directly affect various tissues and metabolic functions.

In conclusion, the present data show that 2'-FL supplementation can improve gut barrier integrity and vagally-mediated signaling to improve appetite control leading to a decrease in food intake. These effects were associated with compositional changes in the gut microbiota and microbial metabolites. The downstream metabolic benefits seen in liver and adipose tissue may be due to decreased food intake or may be mediated by systemic effects of 2'-FL or microbial metabolites directly at the tissue level. Like all studies aimed at interrogating the consequences of dietary supplementation, prebiotics in particular, on both metabolism and gut microbiota/metabolites, the conclusion from the analyses must inherently be correlative. The correlations form the basis for future experimental investigation designed to explore causality. These findings support the application of 2 -FL in the control of gut barrier function and metabolic homeostasis under normal and pathophysiological conditions.

\section{Author contributions}

Conceptualization: HER, DB, MG; data curation: SL, MG, JR, $\mathrm{KK}, \mathrm{MB}, \mathrm{DB}$; formal analysis: SL, MG, WV, KK, MB; funding acquisition: HER, DB; investigation: SL, MG, WV, JR, KK, MB; methodology: SL, MG, MB, DB, HER; project administration: SL, MG, DB, HER; resources: HER, DB; software: n/a; supervision: SL, MG, DB, HER; validation: SL, MG, KK, MB, DB; visualization: SL, MG, KK, JR, MB; writing - original draft: SL; writing - review \& editing: SL, MG, JR, KK, MB, DB, HER.

\section{Conflicts of interest}

HER, MG, SL, WV, MB have no financial or personal, or otherwise conflicts of interests. DB is the co-founder of Evolve Biosystems, a company focused on probiotics.

\section{Acknowledgements}

We would like to acknowledge the UC Davis MMPC Cores: Gut Microbiota and Host Response and Energy Balance, Exercise, \& Behavior (NIH grant DK41004), BASF and Dr Carlito Lebrilla lab (Dept of Chemistry, UC Davis) for technical support for this study.

\section{References}

1 M. Romaní-Pérez, A. Agusti and Y. Sanz, Innovation in microbiome-based strategies for promoting metabolic health, Curr. Opin. Clin. Nutr. Metab. Care, 2017, 20, 484491.

2 S. Carding, K. Verbeke, D. T. Vipond, B. M. Corfe and L. J. Owen, Dysbiosis of the gut microbiota in disease, Microb. Ecol. Health Dis., 2015, 26, 26191.

3 J. F. Cryan and S. M. O'Mahony, The microbiome-gut-brain axis: from bowel to behavior, Neurogastroenterol. Motil., 2011, 23, 187-192.

4 A. C. Vaughn, E. M. Cooper, P. M. DiLorenzo, L. J. O'Loughlin, M. E. Konkel, J. H. Peters, et al., Energydense diet triggers changes in gut microbiota, reorganization of gut-brain vagal communication and increases body fat accumulation, Acta Neurobiol. Exp., 2017, 77, 1830.

5 S. Lee, K. I. Keirsey, R. Kirkland, Z. I. Grunewald, J. G. Fischer and C. B. de La Serre, Blueberry Supplementation Influences the Gut Microbiota, Inflammation, and Insulin Resistance in High-Fat-Diet-Fed Rats, J. Nutr., 2018, 148, 209-219.

6 S. Lee, R. Kirkland, Z. I. Grunewald, Q. Sun, L. Wicker and C. B. de La Serre, Beneficial Effects of Non-Encapsulated or Encapsulated Probiotic Supplementation on Microbiota Composition, Intestinal Barrier Functions, Inflammatory Profiles, and Glucose Tolerance in High Fat Fed Rats, Nutrients, 2019, 11, 1975-1991.

7 S. Lee, M. L. Goodson, W. Vang, K. Kalanetra, D. Barile and H. Raybould, 2'-fucosyllactose Supplementation Improves Gut-Brain Signaling and Diet-Induced Obese Phenotype and Changes the Gut Microbiota in High Fat-Fed Mice, Nutrients, 2020, 12, 1003-1020. 
8 S. Wang, X.-F. Huang, P. Zhang, H. Wang, Q. Zhang, S. Yu, et al., Chronic rhein treatment improves recognition memory in high-fat diet-induced obese male mice, J. Nutr. Biochem., 2016, 36, 42-50.

9 S. Wang, X.-F. Huang, P. Zhang, K. A. Newell, H. Wang, K. Zheng, et al., Dietary teasaponin ameliorates alteration of gut microbiota and cognitive decline in diet-induced obese mice, Sci. Rep., 2017, 7, 12203.

10 X. Yang, M. Zheng, S. Hao, H. Shi, D. Lin, X. Chen, et al., Curdlan Prevents the Cognitive Deficits Induced by a HighFat Diet in Mice via the Gut-Brain Axis, Front. Neurosci., 2020, 14, 384.

11 J. Sun, S. Liu, Z. Ling, F. Wang, Y. Ling, T. Gong, et al., Fructooligosaccharides Ameliorating Cognitive Deficits and Neurodegeneration in APP/PS1 Transgenic Mice through Modulating Gut Microbiota, J. Agric. Food Chem., 2019, 67, 3006-3017.

12 S. Filosa, F. Di Meo and S. Crispi, Polyphenols-gut microbiota interplay and brain neuromodulation, Neural Regener. Res., 2018, 13, 2055-2059.

13 M. Roberfroid, G. R. Gibson, L. Hoyles, A. L. McCartney, R. Rastall, I. Rowland, et al., Prebiotic effects: metabolic and health benefits, Br. J. Nutr., 2010, 104(Suppl), S1-S63.

14 F. De Vadder, P. Kovatcheva-Datchary, D. Goncalves, J. Vinera, C. Zitoun, A. Duchampt, et al., Microbiota-generated metabolites promote metabolic benefits via gut-brain neural circuits, Cell, 2014, 156, 84-96.

15 E. M. Quinn, L. Joshi and R. M. Hickey, Symposium review: Dairy-derived oligosaccharides-Their influence on hostmicrobe interactions in the gastrointestinal tract of infants, J. Dairy Sci., 2020, 103, 3816-3827.

16 R. M. Erney, W. T. Malone, M. B. Skelding, A. A. Marcon, K. M. Kleman-Leyer, M. L. O'Ryan, et al., Variability of human milk neutral oligosaccharides in a diverse population, J. Pediatr. Gastroenterol. Nutr., 2000, 30, 181-192.

17 C. Kong, M. M. Faas, P. de Vos and R. Akkerman, Impact of dietary fibers in infant formulas on gut microbiota and the intestinal immune barrier, Food Funct., 2020, 11, 94459467.

18 N. Kirmiz, R. C. Robinson, I. M. Shah, D. Barile and D. A. Mills, Milk Glycans and Their Interaction with the Infant-Gut Microbiota, Annu. Rev. Food Sci. Technol., 2018, 9, 429-450.

19 S. Karav, A. Le Parc, J. M. L. N. de Moura Bell, S. A. Frese, N. Kirmiz, D. E. Block, et al., Oligosaccharides Released from Milk Glycoproteins Are Selective Growth Substrates for Infant-Associated Bifidobacteria, Appl. Environ. Microbiol., 2016, 82, 3622-3630.

20 G. M. Ruiz-Palacios, L. E. Cervantes, P. Ramos, B. ChavezMunguia and D. S. Newburg, Campylobacter jejuni binds intestinal $\mathrm{H}(\mathrm{O})$ antigen (Fuc alpha 1, 2Gal beta 1, 4GlcNAc), and fucosyloligosaccharides of human milk inhibit its binding and infection, J. Biol. Chem., 2003, 278, 14112-14120.

21 Z.-T. Yu, N. N. Nanthakumar and D. S. Newburg, The Human Milk Oligosaccharide 2'-Fucosyllactose Quenches
Campylobacter jejuni-Induced Inflammation in Human Epithelial Cells HEp-2 and HT-29 and in Mouse Intestinal Mucosa, J. Nutr., 2016, 146, 1980-1990.

22 E. Oliveros, M. Ramirez, E. Vazquez, A. Barranco, A. Gruart, J. M. Delgado-Garcia, et al., Oral supplementation of 2'fucosyllactose during lactation improves memory and learning in rats, J. Nutr. Biochem., 2016, 31, 20-27.

23 H. Matthies, S. Staak and M. Krug, Fucose and fucosyllactose enhance in vitro hippocampal long-term potentiation, Brain Res., 1996, 725, 276-280.

24 E. Vázquez, A. Barranco, M. Ramirez, A. Gruart, J. M. Delgado-Garcia, M. L. Jimenez, et al., Dietary 2'Fucosyllactose Enhances Operant Conditioning and LongTerm Potentiation via Gut-Brain Communication through the Vagus Nerve in Rodents, PLoS One, 2016, 11, e0166070.

25 M. K. Hamilton and H. E. Raybould, Bugs, guts and brains, and the regulation of food intake and body weight, Int. J. Obes. Suppl., 2016, 6, S8-S14.

26 P. D. Cani, M. Van Hul, C. Lefort, C. Depommier, M. Rastelli and A. Everard, Microbial regulation of organismal energy homeostasis, Nat. Metab., 2019, 1, 34-46.

27 G. de Lartigue, C. B. de La Serre and H. E. Raybould, Vagal afferent neurons in high fat diet-induced obesity; intestinal microflora, gut inflammation and cholecystokinin, Physiol. Behav., 2011, 105, 100-105.

28 J. E. Ayala, V. T. Samuel, G. J. Morton, S. Obici, C. M. Croniger, G. I. Shulman, et al., Standard operating procedures for describing and performing metabolic tests of glucose homeostasis in mice, Dis. Model. Mech., 2010, 3, 525-534.

29 D. C. Hoaglin and B. Iglewicz, Fine-tuning some resistant rules for outlier labeling, J. Am. Stat. Assoc., 1987, 82, 11471149.

30 K. J. Livak and T. D. Schmittgen, Analysis of relative gene expression data using real-time quantitative PCR and the 2 (-Delta Delta C(T)) Method, Methods, 2001, 25, 402-408.

31 S. A. Frese, K. Parker, C. C. Calvert and D. A. Mills, Diet shapes the gut microbiome of pigs during nursing and weaning, Microbiome, 2015, 3, 28.

32 C. Lozupone, M. E. Lladser, D. Knights, J. Stombaugh and R. Knight, UniFrac: an effective distance metric for microbial community comparison, ISME J., 2011, 5, 169172.

33 E. Bolyen, J. R. Rideout, M. R. Dillon, N. A. Bokulich, C. C. Abnet, G. A. Al-Ghalith, et al., Reproducible, interactive, scalable and extensible microbiome data science using QIIME 2, Nat. Biotechnol., 2019, 37, 852-857.

34 B. J. Callahan, P. J. McMurdie, M. J. Rosen, A. W. Han, A. J. A. Johnson and S. P. Holmes, DADA2: High-resolution sample inference from Illumina amplicon data, Nat. Methods, 2016, 13, 581-583.

35 N. Segata, J. Izard, L. Waldron, D. Gevers, L. Miropolsky, W. S. Garrett, et al., Metagenomic biomarker discovery and explanation, Genome Biol., 2011, 12, R60.

36 F. Rivera-Chávez, L. F. Zhang, F. Faber, C. A. Lopez, M. X. Byndloss, E. E. Olsan, et al., Depletion of Butyrate- 
Producing Clostridia from the Gut Microbiota Drives an Aerobic Luminal Expansion of Salmonella, Cell Host Microbe, 2016, 19, 443-454.

37 L. R. Ruhaak, C. Stroble, M. A. Underwood and C. B. Lebrilla, Detection of milk oligosaccharides in plasma of infants, Anal. Bioanal. Chem., 2014, 406, 5775-5784.

38 L. V. Hooper, M. H. Wong, A. Thelin, L. Hansson, P. G. Falk and J. I. Gordon, Molecular analysis of commensal host-microbial relationships in the intestine, Science, 2001, 291, 881-884.

39 R. E. Ley, F. Bäckhed, P. Turnbaugh, C. A. Lozupone, R. D. Knight and J. I. Gordon, Obesity alters gut microbial ecology, Proc. Natl. Acad. Sci. U. S. A., 2005, 102, 11070-11075.

40 F. Fåk, G. Jakobsdottir, E. Kulcinskaja, N. Marungruang, C. Matziouridou, U. Nilsson, et al., The physico-chemical properties of dietary fibre determine metabolic responses, short-chain Fatty Acid profiles and gut microbiota composition in rats fed low- and high-fat diets, PLoS One, 2015, 10, e0127252.

41 Z.-T. Yu, C. Chen and D. S. Newburg, Utilization of major fucosylated and sialylated human milk oligosaccharides by isolated human gut microbes, Glycobiology, 2013, 23, 12811292.

42 J. K. Nicholson, E. Holmes, J. Kinross, R. Burcelin, G. Gibson, W. Jia, et al., Host-gut microbiota metabolic interactions, Science, 2012, 336, 1262-1267.

43 Y.-S. Lee, T.-Y. Kim, Y. Kim, S.-H. Lee, S. Kim, S. W. Kang, et al., Microbiota-Derived Lactate Accelerates Intestinal Stem-Cell-Mediated Epithelial Development, Cell Host Microbe, 2018, 24, 833-846.e6.

44 P. Ranganathan, A. Shanmugam, D. Swafford, A. Suryawanshi, P. Bhattacharjee, M. S. Hussein, et al., GPR81, a Cell-Surface Receptor for Lactate, Regulates Intestinal Homeostasis and Protects Mice from Experimental Colitis, J. Immunol., 2018, 200, 1781-1789.

45 R. Singh, S. Chandrashekharappa, S. R. Bodduluri, B. V. Baby, B. Hegde, N. G. Kotla, et al., Enhancement of the gut barrier integrity by a microbial metabolite through the Nrf2 pathway, Nat. Commun., 2019, 10, 89.

46 G. de Lartigue, Role of the vagus nerve in the development and treatment of diet-induced obesity, J. Physiol., 2016, 594, 5791-5815.

47 L. A. Zenewicz, G. D. Yancopoulos, D. M. Valenzuela, A. J. Murphy, S. Stevens and R. A. Flavell, Innate and adaptive interleukin-22 protects mice from inflammatory bowel disease, Immunity, 2008, 29, 947-957.

48 A. Biddle, L. Stewart, J. Blanchard and S. Leschine, Untangling the Genetic Basis of Fibrolytic Specialization by
Lachnospiraceae and Ruminococcaceae in Diverse Gut Communities, Diversity, 2013, 5, 627-640.

49 K. P. Scott, S. H. Duncan and H. J. Flint, Dietary fibre and the gut microbiota, Nutr. Bull., 2008, 33, 201-211.

50 R. L. Mendez, C. Miranda, C. R. Armour, T. J. Sharpton, J. F. Stevens and J. Y. Kwon, Supplementation with Sea Vegetables Palmaria mollis and Undaria pinnatifida Exerts Metabolic Benefits in Diet-Induced Obesity in Mice, Curr. Dev. Nutr., 2020, 4, nzaa072.

51 P. Zhang, Y. Yu, Y. Qin, Y. Zhou, R. Tang, Q. Wang, et al., Alterations to the microbiota-colon-brain axis in high-fatdiet-induced obese mice compared to diet-resistant mice, J. Nutr. Biochem., 2019, 65, 54-65.

52 J. C. d'Avila, L. D. Siqueira, A. Mazeraud, E. P. Azevedo, D. Foguel, H. C. Castro-Faria-Neto, et al., Age-related cognitive impairment is associated with long-term neuroinflammation and oxidative stress in a mouse model of episodic systemic inflammation, J. Neuroinflammation, 2018, $15,28$.

53 M. Longo, F. Zatterale, J. Naderi, L. Parrillo, P. Formisano, G. A. Raciti, et al., Adipose Tissue Dysfunction as Determinant of Obesity-Associated Metabolic Complications, Int. J. Mol. Sci., 2019, 20, 2358.

54 Z. Song, A. M. Xiaoli and F. Yang, Regulation and Metabolic Significance of De Novo Lipogenesis in Adipose Tissues, Nutrients, 2018, 10, 1383.

55 C. Saponaro, M. Gaggini, F. Carli and A. Gastaldelli, The Subtle Balance between Lipolysis and Lipogenesis: A Critical Point in Metabolic Homeostasis, Nutrients, 2015, 7, 9453-9474.

56 R. Li, H. Wang, Q. Shi, N. Wang, Z. Zhang, C. Xiong, et al., Effects of oral florfenicol and azithromycin on gut microbiota and adipogenesis in mice, PLoS One, 2017, 12, e0181690.

57 S. Rudloff, G. Pohlentz, C. Borsch, M. J. Lentze and C. Kunz, Urinary excretion of in vivo ${ }^{13} \mathrm{C}$-labelled milk oligosaccharides in breastfed infants, Br. J. Nutr., 2012, 107, 957-963.

58 S. Rudloff, G. Pohlentz, L. Diekmann, H. Egge and C. Kunz, Urinary excretion of lactose and oligosaccharides in preterm infants fed human milk or infant formula, Acta Paediatr., 1996, 85, 598-603.

59 A. Santos-Fandila, A. Zafra-Gómez, E. Vazquez, A. Navalón, R. Rueda and M. Ramírez, Ultra high performance liquid chromatography-tandem mass spectrometry method for the determination of soluble milk glycans in rat serum, Talanta, 2014, 118, 137-146. 